\title{
Clinically node negative breast cancer patients undergoing breast conserving therapy, sentinel lymph node procedure versus follow-up: a Dutch randomized controlled multicentre trial (BOOG 2013-08)
}

\author{
L. M. van Roozendaal ${ }^{1,2+}$, M. L. G. Vane ${ }^{1,2^{*}+}$, T. van Dalen ${ }^{3}$, J. A. van der Hage ${ }^{4}$, L. J. A. Strobbe ${ }^{5}$, L. J. Boersma ${ }^{2,6}$, \\ S. C. Linn ${ }^{7}$, M. B. I. Lobbes ${ }^{8}$, P. M. P. Poortmans ${ }^{9}$, V. C. G. Tjan-Heijnen ${ }^{2,10}$, K. K. B. T. Van de Vijver ${ }^{11}$, J. de Vries ${ }^{12}$, \\ A. H. Westenberg ${ }^{13}$, A. G. H. Kessels ${ }^{14}$, J. H. W. de Wilt ${ }^{15}$ and M. L. Smidt ${ }^{1,2}$
}

\begin{abstract}
Background: Studies showed that axillary lymph node dissection can be safely omitted in presence of positive sentinel lymph node(s) in breast cancer patients treated with breast conserving therapy. Since the outcome of the sentinel lymph node biopsy has no clinical consequence, the value of the procedure itself is being questioned. The aim of the BOOG 2013-08 trial is to investigate whether the sentinel lymph node biopsy can be safely omitted in clinically node negative breast cancer patients treated with breast conserving therapy.

Methods: The BOOG 2013-08 is a Dutch prospective non-inferiority randomized multicentre trial. Women with pathologically confirmed clinically node negative T1-2 invasive breast cancer undergoing breast conserving therapy will be randomized for sentinel lymph node biopsy versus no sentinel lymph node biopsy. Endpoints include regional recurrence after 5 (primary endpoint) and 10 years of follow-up, distant-disease free and overall survival, quality of life, morbidity and cost-effectiveness. Previous data indicate a 5 -year regional recurrence free survival rate of $99 \%$ for the control arm and $96 \%$ for the study arm. In combination with a non-inferiority limit of 5\% and probability of 0.8 , this result in a sample size of 1.644 patients including a lost to follow-up rate of $10 \%$. Primary and secondary endpoints will be reported after 5 and 10 years of follow-up.
\end{abstract}

Discussion: If the sentinel lymph node biopsy can be safely omitted in clinically node negative breast cancer patients undergoing breast conserving therapy, this study will cost-effectively lead to a decreased axillary morbidity rate and thereby improved quality of life with non-inferior regional control, distant-disease free survival and overall survival.

Trial registration: The BOOG 2013-08 study is registered in ClinicalTrials.gov since October 20, 2014, Identifier: NCT02271828. https://clinicaltrials.gov/ct2/show/NCT02271828

Keywords: Breast neoplasms, Breast cancer, Sentinel lymph node biopsy, Breast conserving therapy

\footnotetext{
* Correspondence: marissa.vane@mumc.nl

${ }^{\dagger}$ Equal contributors

'Division of Surgical Oncology, Maastricht University Medical Centre, P.O. Box

58006202 AZ Maastricht, The Netherlands

${ }^{2}$ GROW - School for Oncology and Developmental Biology, Maastricht

University Medical Centre, Maastricht, the Netherlands

Full list of author information is available at the end of the article
} 


\section{Background}

More than fifteen years ago, the sentinel lymph node biopsy (SLNB) was introduced in clinically node negative breast cancer patients to evaluate their lymph node status for diagnostic purposes. In case of a negative sentinel lymph node (SLN) an axillary lymph node dissection (ALND) was omitted. The SLN is negative in approximately $74 \%$ of patients in a general breast cancer population [1, 2]. Although SLNB is less invasive compared to ALND, short-term complications still occur in $25 \%$ of the patients. Most reported complications are axillary seroma, wound infections, hematoma, anaphylactic reaction, axillary paresthesia, and lymphedema, which is described in $8 \%$ of patients after a follow-up of only 3 years, resulting in significant reduction of quality of life (QoL) of breast cancer survivors [3-7].

Ever since the National Surgical Adjuvant Breast and Bowel Project (NSABP B-04) trial, the need for completion axillary treatment for clinically node negative patients has been questioned. This trial revealed that omitting ALND in clinically node negative patients did not affect disease-free survival (DFS) and overall survival (OS) [8]. Patients were randomized for mastectomy-only, mastectomy with ALND or mastectomy with axillary radiotherapy (RT). About $40 \%$ of the patients who underwent mastectomy-only had lymph node metastases that were not removed at the time of initial surgery. During followup, ipsilateral lymph nodes became clinically apparent in less than half of these patients (18.6\%). Nevertheless, omitting ALND in clinically node negative patients did not affect DFS and OS, even after 25 years of follow-up and without adjuvant RT or systemic therapy.

The more recent American College of Surgeons Oncology Group (ACOSOG) Z0011 and International Breast Cancer Study Group (IBCSG) 23-01 trials investigated whether completion ALND can be safely omitted in patients with a metastasis in the SLN. The ACOSOG Z0011 trial included patients with 1-2 macrometastatic SLN(s) who were treated with breast conserving therapy (BCT) [9]. The IBCSG 23-01 trial only included patients with a micrometastasis in the SLN, but gave no restriction on type of breast surgery [10]. Most patients were treated with adjuvant systemic treatment (both 97\%). Patients in these trials were randomized to completion ALND or watchful waiting. Additional lymph node metastases beyond the SLN were detected in 27\% (ACOSOG Z0011) and 11\% (IBCSG 23-01) in the ALND groups $[9,10]$. Despite the fact that nodal metastases remained in situ in a considerable percentage of patients in the 'watchful waiting' groups, omitting completion ALND did not result in inferior regional recurrence (RR) rates, DFS and OS after 5-years of follow-up. These studies indicated that completion ALND can be safely omitted in presence of positive $\operatorname{SLN}(\mathrm{s})$ in patients treated with $\mathrm{BCT}$ and adjuvant systemic treatment. Since the outcome of the SLNB has no clinical consequence, the value of the SLNB itself is being questioned.

Clinically node negative status in the NSABP B-04, ACOSOG Z0011 and IBCSG 23-01 trials was based on negative physical examination of the axilla. Preoperative nodal staging with physical examination has a low accuracy, with a sensitivity of only $32 \%$ [11-14]. In the Netherlands, axillary ultrasound is part of standard preoperative axillary work-up. The sensitivity of axillary ultrasound (in combination with tissue sampling where deemed necessary) is approximately $80 \%$ [15]. Furthermore, a negative axillary ultrasound excludes the presence of four or more lymph node metastases, with a negative predictive value of $93-96 \%$ in the general breast cancer population [16-18]. Therefore, axillary ultrasound improves preoperative selection of node negative patients, as it selects patients with a more favourable tumour load and confidently excludes advanced nodal disease.

Several factors besides surgery have proven to decrease $\mathrm{RR}$ rates. For instance, it is assumed that biology plays an important role in dormancy of nodal metastases. Less than half of the patients with occult nodal metastases in the NSABP B-04 trial, developed clinically detectable lymph nodes during follow-up, none of these patients received adjuvant systemic or RT [8]. Adjuvant systemic therapy is known to decrease RR rates [19]. Primary systemic therapy can eradicate lymph node metastases with a reported pathologic complete response rates of $20-40 \%$ [20-23]. Lack of knowledge on the pathological lymph node status is nowadays hardly influencing systemic therapy indication [24]. Low RR rates in the ACOSOG Z0011 (and IBCSG 23-01) trial might be due to whole breast irradiation (WBI) following lumpectomy [25-28]. RT of the breast may contribute to the elimination of (occult) lymph node metastases by including part of the axilla [25]. A recent study has shown that, even with contemporary 3D radiation techniques, the SLN receives an elective radiation dose in $76 \%$ of patients [29]. Biology, adjuvant systemic and RT most likely diminish the risk that possible lymph node metastases left in situ develop into clinically detectable lymph nodes.

This randomized controlled BOOG 2013-08 trial proposes to demonstrate that the SLNB can be safely omitted in breast cancer patients with a clinically node negative T1-2 status undergoing BCT. This trial aims to decrease the number of breast cancer patients receiving an invasive axillary procedure, to decrease the axillary morbidity rate, thereby improving QoL and reducing the costs of SLNB without affecting regional control and survival.

\section{Main study objectives}

Primary objective of this study is to investigate whether watchful waiting (i.e. no SLNB) is not inferior in terms of 5 and 10-year RR rate to the current axillary staging 
regimen in breast cancer patients with a clinically node negative T1-2 status undergoing BCT. Secondary objectives are distant-DFS, OS, local recurrence (LR) rate, contralateral breast cancer, number of delayed axillary treatment, adjuvant RT, QoL, axillary morbidity rate, and cost-effectiveness after 5- and 10-years of follow-up.

\section{Methods}

\section{Study design}

The BOOG 2013-08 is a Dutch prospective noninferiority randomized controlled multicentre trial. Women with pathologically confirmed unilateral clinically node negative $\mathrm{T} 1-2$ invasive breast cancer undergoing $\mathrm{BCT}$ are randomized to SLNB or watchful waiting (i.e. no SLNB). Primary and secondary endpoints will be reported after 5 and 10 years of follow-up. The BOOG 2013-08 is a multicentre trial and will be performed in 35 participating centres (Table 1). This study design was based on the BOOG 2013-07 trial of the same research group [30].

\section{Study population}

Women $\geq 18$ years with pathologically confirmed clinically node negative T1-2 invasive breast cancer, treated with BCT (lumpectomy and WBI) are eligible for inclusion. Clinically node negative is defined as no signs of axillary lymph node metastases, consisting of a negative physical examination of the axilla and preoperative axillary ultrasound (or negative cyto-/histopathology in case of a suspicious axillary lymph node) [30].

Exclusion criteria are: metastatic disease; bilateral breast cancer; history of invasive breast cancer; previous surgical treatment or RT of the ipsilateral axilla (except surgery for superficially skin lesions, such as naevi or hidradenitis suppurativa); other prior malignancies, except successfully treated malignancies $>5$ years before inclusion, successfully treated basal cell and squamous cell skin cancer, and carcinoma in situ of the ipsilateral, contralateral breast or cervix; and pregnancy or lactation [30]. Primary systemic therapy and breast reconstructions are no exclusion criteria.

\section{Axillary ultrasound}

In the Netherlands, axillary ultrasound is standard care for preoperative nodal staging of breast cancer patients [30]. The following criteria are used to identify axillary lymph node metastases during an axillary ultrasound: cortical thickening, long to short axis ratio of $<2$ (i.e. round), effacement or replacement of the fatty hilum, and/or nonhilar blood flow [30]. If cortical thickening is $>2.3 \mathrm{~mm}$, fine-needle aspiration biopsy is performed [31]. Further, the radiologist can make a subjective assessment of cortical thickening during real-time imaging $[16,32,33]$. When suspicious lymph nodes are observed during an axillary ultrasound, fine-needle aspiration
Table 1 Participating centres BOOG 2013-08 study

Name participating centre

1. Alrijne hospital, Alphen aan de Rijn and Leiden

2. Amphia hospital, Breda

3. Antonius hospital, Sneek

4. Bronovo hospital and Medical Centre Haaglanden, den Haag

5. Canisius-Wilhelmina hospital, Nijmegen

6. Catharina hospital, Eindhoven

7. Deventer hospital, Deventer

8. Diakonessenhuis, Utrecht

9. Flevoziekenhuis, Almere

10. Gelderse Vallei, Ede

11. Gelre hospital, Apeldoorn

12. Groene Hart hospital, Gouda

13. Haga hospital, den Haag

14. Isala Clinic, Zwolle

15. Isala Diaconessenhuis, Meppel

16. Jeroen Bosch hospital, den Bosch

17. Laurentius hospital, Roermond

18. Maastricht University Medical Centre, Maastricht

19. Martini hospital, Groningen

20. Maxima Medical Centre, Eindhoven and Veldhoven

21. Meander Medical Centre, Amersfoort

22. Medical Spectrum Twente, Enschede and Oldenzaal

23. Netherlands Cancer Institute - Antoni van Leeuwenhoek, Amsterdam

24. Noordwest ZHG, Alkmaar

25. Radboud University Medical Centre, Nijmegen

26. Reinier de Graaf Groep, Delft

27. Rijnstate hospital, Arnhem

28. Rivierenland hospital, Tiel

29. Sint Antonius hospital, Utrecht and Nieuwegein

30. Sint Elisabeth hospital, Tilburg

31. Spaarne Gasthuis, Haarlem and Hoofddorp

32. University Medical Centre Groningen, Groningen

33. University Medical Centre Utrecht, Utrecht

34. Zuyderland hospital Sittard and Heerlen

35. Zuwehofpoort hospital, Woerden

cytology or core needle biopsy is recommended. If more than one suspicious lymph node is present, the most suspicious lymph node is sampled [30].

\section{Breast conserving therapy}

$\mathrm{BCT}$ is defined as lumpectomy followed by WBI. The primary tumour size is determined during pathological assessment. Immunohistochemical (IHC) staining is used to determine the hormone receptor status and is 
considered positive if $\geq 10 \%$ of the cells stain positive. HER2neu status is determined by IHC, or in case of $2+$ by Chromogenic In Situ Hybridization (CISH) or Fluorescence In Situ Hybridization (FISH) [30].

World Health Organization is used to define the histological tumour type. The modified Bloom-Richardson grading system is used to assess histological tumour grading. Multifocality is defined as foci or carcinoma separate from the primary breast tumor. Lymphovascular invasion is defined as $\geq 1$ tumour cells in a lymphatic or vascular structure [30].

\section{Sentinel lymph node biopsy}

The SLN procedure is performed using technetium-99 m Nanocolloid as a radioactive tracer and blue dye for lymphatic mapping. Both are injected into breast parenchymal tissue surrounding the tumour, biopsy cavity or periareolar. The SLN is identified using the following triple technique: lymphoscintigraphy, intraoperative use of the gamma probe, and intraoperative detection of the blue lymphatic vessels. After removal of the $\operatorname{SLN}(\mathrm{s})$, palpation of the axilla is performed to identify and remove additional suspicious (non-) SLN(s) [30].

Each SLN is examined at three histological levels (500$\mu \mathrm{m}$ intervals) as a minimal requirement for pathological assessment. Two parallel sections on each level are performed, one for haematoxylin and eosin (H\&E) staining and one for IHC staining [30]. When H\&E staining is negative IHC staining is done. Lymph nodes marked by the surgeon as non-SLNs are also examined with $\mathrm{H} \& \mathrm{E}$ and if negative with cytokeratin IHC staining. The diameter of each metastasis and the presence of extranodal growth must be determined. Isolated tumour cells $(<0.2 \mathrm{~mm})$ are considered as SLN negative [30].

\section{Radiation therapy}

\section{Dose and fractionation for whole breast radiation}

A fractionation scheme equivalent to $25 \times 2$ Gray (Gy), 5 fractions per week is applied. Most Dutch RT centers use a scheme of 15-16 × 2.67 Gy, 5 fractions per week. In case of focal irradical resection, a higher boost dose is recommended (equivalent to $10-13 \times 2$ Gy) [30]. Partial breast irradiation is not allowed.

\section{Delineation of whole breast radiation}

The European Society for Radiotherapy and Oncology (ESTRO) guidelines of Offersen et al. are used to perform delineation of target volumes and organs at risk [34]. Delineation of tumour bed, including a clinical target volume (CTV) and a planning target volume (PTV) is obligatory. In addition, delineation of axillary nodal regions, axilla level 1, 2, Rotter nodes and 3 is obligatory, even when there is no indication for axillary radiation.
In case of left-sided breast cancer, delineation of the heart and lungs is obligatory [30].

\section{Radiation technique and dose distribution}

The dose in the target volume (whole breast, with or without axillary and periclavicular nodes irradiation) must be between $95 \%-107 \%$ of the prescribed dose [30]. The Central Lung Distance must be $<3 \mathrm{~cm}$ (in case of tangential fields) and mean lung dose should be $<7.5$ Gy. The Maximum Heart Distance (in case of tangential fields) must be $<1 \mathrm{~cm}$, and the heart volume receiving $>10$ Gy should be $<15 \%$. If lung or heart constraints cannot be met, some underdose in the breast can be accepted to reach the constraints, provided that the PTV of the tumour bed is adequately covered. Breath holding techniques to reduce heart dose are highly recommended for left sided breast cancer patients. For evaluation purposes, the minimum, maximum and mean dose of the axilla level 1, 2, Rotter nodes, and 3 must be recorded [30].

\section{Consent and randomization}

Eligible patients will be informed about the study aim, randomization procedure, consequences of participating (i.e. possible adverse events), and their rights and responsibilities by the attending surgeon. Written informed consent must be obtained and randomization will be performed preoperatively. Patients will be randomized between SLNB (control arm) and no SLNB (study arm).

Patients will be stratified by: clinical tumour size $(<3 \mathrm{~cm}$ vs. $\geq 3 \mathrm{~cm}$ ), grading (grade I-II vs. III), oestrogen receptor status (positive vs. negative), HER2neu status (positive vs. negative), age ( $\leq 50,50 \leq 75,>75$ years), primary systemic therapy (yes vs. no) and participating centre.

\section{Systemic therapy}

According to the Dutch breast cancer guideline and multidisciplinary approach, indication for systemic therapy is determined for each individual patient [30, 31]. Adjuvant! Online can be used to estimate the 10-years breast cancer specific survival and the risk reduction by systemic therapy, with or without knowledge of the pathological nodal status. Validated gene expression profiling can be used as an addition to clinicopathologic characteristics, in case of doubt about the indication for adjuvant systemic therapy based on the traditional prognostic factors. Primary systemic therapy is allowed, if the patient has a clinically node negative T1-2 status that is amenable to BCT surgery pre-systemic therapy.

\section{Follow-up}

The first five years of follow-up consists of outpatient clinic visits once yearly, including a physical examination of the axilla and a full-field digital mammography (FFDM). Year six to ten of follow-up, consists of a FFDM 
annually in patients aged $\leq 60$ years or once every two years in patients aged $>60$ years [30]. Additional diagnostic imaging is only performed on indication. Axillary ultrasound is performed, in case of a clinical suspicion of axillary lymph node metastases. Staging for distant metastatic disease is performed, if an axillary lymph node metastasis is confirmed (cyto-/histopathology) or in case of a clinical suspicion of distant metastatic disease [30].

\section{Quality of life}

A Dutch version of two validated QoL questionnaires of the European Organization for the Research and Treatment of Cancer (EORTC QLQ-C30 and QLQ-BR 23) are used to assess the QoL of breast cancer patients. To assess the subjective morbidity the validated Lymphedema Functioning, Disability and Health questionnaire (Lymph-ICF) is used. A validated short version of the Spielberger State-Trait Anxiety Inventory (STAI-trait) and Neuroticism Extraversion Openness Five Factor Inventory (NEO-FFI) is used to measure if anxiety and personality traits influences the outcome of QoL [3538]. The combination of these questionnaires will provide information on the general and breast cancer specific QoL, subjective morbidity, and anxiety and personality traits that might influence the outcome of QoL [39]. The first QoL questionnaires are provided pre-randomisation for baseline measurement, and the following are provided post-randomization at six months, and at 1, 2, 3, 5 and 10 years. Patients are eligible for evaluation when at least the pre-randomisation questionnaire and the subsequent questionnaire are completed [30].

\section{Adverse events}

Any undesirable experience during the study, whether or not considered related to the protocol treatment is defined as an adverse events (AEs), including seroma, postoperative haemorrhage, wound complication/infection, lymphedema of the arm or chest wall, neuralgia, paraesthesia, decreased arm or shoulder motion, muscle weakness of the arm or shoulder, and pain in the arm or shoulder. NCI/CTCAE 4.0 grading criteria is used to grade the severity of the AE into mild, moderate, or severe, in combination with the degree of limitation in activities of daily living [30].

An untoward medical occurrence or effect related to protocol treatment that results in death, hospitalisation or prolongation of existing inpatients hospitalisation, or surgery is defined as a serious adverse event (SAE). Protocol treatment is defined as $\mathrm{BCT}$ of the primary tumour, WBI, SLNB or completion axillary treatment. Any other operation or adjuvant treatment is not considered protocol treatment [30].
The local investigator of the participating centre is responsible for reporting the SAE to the central data centre within $24 \mathrm{~h}$. The principal investigators of the study are responsible for SAE assessment and reporting to the accredited medical ethics committee within 15 days. For fatal or life threatening cases, the term is maximal 7 days for a preliminary report with another 8 days to completion the report. All SAEs will be followed until they have abated, or until a stable situation has been reached. Depending on the event, followup may require additional tests or medical procedures as indicated and/or referral to the general physician or a medical specialist [30].

\section{Statistics \\ Endpoints}

Primary endpoint of this study is RR rate after 5 and 10years of follow-up. Secondary endpoints are regional recurrence free survival (RRFS), number of delayed axillary treatment, distant-DFS, OS, LR rate, other-RR rate, contralateral breast cancer rate, diagnosis of recurrence outside the axillary region, percentage difference in the administration of postoperative RT, axillary morbidity rate, QoL and cost-effectiveness after 5- and 10-years of followup. RR, other-RR, LR and distant recurrence are defined according the Maastricht Delphi Consensus on Event Definition by Moossdorff et al. [40, 41]. Pathological confirmation of a RR is mandatory. All suspected lesions for recurrence on imaging, which are not accessible for histology or cytology, are presented to the Data Safety Monitoring Board (DSMB) for an independent review.

Time to event endpoints are defined as the time interval between the date of randomization and the date of first suspicion of the predefined recurrence, or the date of death, whichever comes first, measured in days. Patients in whom recurrence is not observed and are still alive are censored at the date of last follow-up. Death from breast cancer and its treatment, death from a second primary invasive non-breast cancer, and death from other- or an unknown cause are recorded [30].

Administration of (neo) adjuvant systemic therapy is registered and the percentage difference between both study arms is recorded. Axillary morbidity rate is assessed using a validated questionnaire and by predefined AE that are recorded by the treating physician. QoL is assessed using validated questionnaires. Cost-effectiveness is assessed using the EQ-5D health questionnaire [30].

\section{Sample size}

Previous data indicate a 5-year regional recurrence free survival rate of $99 \%$ for the control arm and $96 \%$ for the study arm. The expected regional recurrence free survival rate and non-inferiority limit of $5 \%$ (delta) with a probability of 0.8 , result in a sample size of 747 per arm. 
When taking in account a lost to follow-up rate of $10 \%$, 1.644 patients need to be randomized. An annual accrual of 856 patients can be achieved, when taking into consideration the incidence of women diagnosed with invasive breast cancer in the Netherlands, the rate of patients that is primarily operated (when excluding patients treated systemic therapy only, or patients with metastatic disease and frail elderly), treatment with BCT, the 35 participating hospitals, and an expected accrual rate of $30 \%$. Therefore, two years will suffice to include the 1.644 patients.

\section{Data safety monitoring board}

An independent DSMB is established comprising an independent surgeon, medical oncologist, radiation oncologist and a statistician [30]. The independent DSMB will meet annually to discuss RR, other events, occurrence of AEs, and the percentage difference in administration of adjuvant systemic therapy between both study arms [30]. The DSMB can decide to alter the frequency of discussion. All suspected lesions for recurrence on imaging, which are not accessible for histology or cytology, are presented to the Data Safety Monitoring Board (DSMB) for an independent review. An interim analysis is performed by a statistician, results will be presented to the DSMB for further interpretation. The principal investigators will receive the DSMB recommendations. Should the principle investigators decide not to fully implement the DSMB recommendations, then the principle investigators have to send the recommendation to the accredited medical ethics committee, including a note to substantiate why (part of) this recommendation will not be followed [30].

\section{Stopping rule}

The principle investigators reserved the right to discontinue the study prior to inclusion of the intended number of subjects, but intends only to exercise this right for valid scientific or administrative reasons such as; a negative advice for continuing the study by the DSMB; in case of a percentage difference in the administration of adjuvant systemic therapy of more than $5 \%$ between both study arms; or disappointing accrual so that the total enrolment of 1644 patients seems not feasible [30].

\section{Final analysis}

Per protocol and in the intention to treat population will be used to analyse the primary and secondary endpoints after 5 and 10 years of follow-up. To evaluate the null hypothesis, uncorrected chi-squared statistics will be used. In case of censored data, chi-square test will be based on the Kaplan-Meier estimator. Cox proportional hazards models and Kaplan Meier estimates will be used to analyse the outcome of both groups and to assess the univariable and multivariable association between prognostic variables, treatment and events, using stratification factors. All statistical tests are 1-sided and a $p$ value of 0.05 or less is considered statistically significant [30].

\section{Discussion}

The BOOG 2013-08 trial is a Dutch prospective non-inferiority randomized multicenter trial invesitgating whether the sentinel lymph node biopsy can be safely omitted in clincially node negative breast cancer patients treated with breast conserving therpay. If the sentinel lymph node biopsy can be safely omitted in clinically node negative breast cancer patients undergoing breast conserving therapy, this study will costeffectively lead to a decreased axillary morbidity rate and thereby improved quality of life with non-inferior regional control, distantdisease free survival and overall survival.

\section{Abbreviations}

AE: Adverse event; ALND: Axillary lymph node dissection; BCT: Breast conserving therapy; CISH: Chromogenic In Situ Hybridization; CTV: Clinical target volume; DFS: Disease-free survival; DSMB: Data safety monitoring board; EORTC QLQ-B23: European organization for the research and treatment of cancer quality of life questionnaire - breast cancer module; EORTC QLQ-C30: European organization for the research and treatment of cancer quality of life questionnaire; FFDM: Full field digital mammography; FISH: Fluorescence in situ hybridization; Gy: Gray; H\&E: Haematoxylin and eosin; IHC: Immunohistochemistry; LR: Local recurrence; LymphICF: Lymphedema functioning, disability and health questionnaire; NEOFFI: Neuroticism extraversion openness five factor inventory; OS: Overall survival; PTV: Planning target volume; QoL: Quality of life; RR: Regional recurrence; RRFS: Regional recurrence free survival; RT: Radiotherapy; SAE: Serious adverse event; SLN: Sentinel lymph node; SLNB: Sentinel lymph node biopsy; STAl-trait: State-trait anxiety inventory; WBI: Whole breast irradiation

\section{Acknowledgements}

The authors gratefully acknowledge the contributions of Elise van Leeuwen from the Dutch Breast Cancer Research Group (Borstkanker Onderzoek Groep; BOOG) and Astrid Swinkels, Mariska Stals, Saskia van Gastel, Steffen de Groot, Baukje Hermans, Ria de Peuter and Wendy Dontje from IKNL clinical research department.

\section{Funding}

The BOOG 2013-08 study received grant support from the Dutch Cancer Society for independent data management performed by IKNL clinical research department (KWF-UM 2014-6679), Central Health Insurance (CZ 201400316) and the Netherlands Organization for Health Research and Development (ZONMW 843002624) for salary of independent PhD-students and quality of life questionnaires.

\section{Availability of data and materials} Not applicable.

\section{Authors' contributions}

LVR prepared the manuscript and coordinated the study, MV prepared the manuscript, coordinates the study and is the corresponding author. LVR and MV both contributed equally to this manuscript and should be considered first author. AK, TVD, JvdH, LS, LB, SL, ML, PP, VTH, KVdV, JdV and AW are members of the writing committee. JdW is one of the principal investigators of the study. MS supervised the first authors and is one of the principal investigators of the study. All authors have contributed to study conception and design, have read and edited the manuscript. All authors approved the final manuscript. 


\section{Authors' information}

LVR is surgeon in training and received her PhD degree in surgical oncology at Maastricht University Medical Centre, MV is PhD candidate in surgical oncology at Maastricht University Medical Centre, AK is statistician at Maastricht University Medical Centre, TvD is surgical oncologist at Diakonessenhuis Hospital, JvdH is surgical oncologist at Netherlands Cancer Institute - Antoni van Leeuwenhoek Hospital, LS is surgical oncologist at Canisius-Wilhelmina Hospital, LB is radiation oncologist at Maastricht University Medical Centre (MAASTRO clinic), SL is professor in medical oncology at Netherlands Cancer Institute - Antoni van Leeuwenhoek Hospital, ML is breast radiologist at Maastricht University Medical Centre, PP is professor in radiation oncology at Radboud university medical centre, VTH is professor in medical oncology at Maastricht University Medical Centre, KVdV is pathologist at Netherlands Cancer Institute - Antoni van Leeuwenhoek Hospital, JdV is professor in quality of life in the medical setting at Tilburg University, AW is radiation oncologist at Arnhem Institute for Radiation Oncology, JdW is professor of surgical oncology at Radboud university medical centre, MS is surgical oncologist at Maastricht University Medical Centre.

\section{Ethics approval and consent to participate}

The study is conducted in accordance to the standards of Good Clinical Practice, in agreement with the Declaration of Helsinki and with Dutch law in general and with the Medical Research Involving Human Subjects Act (in Dutch: Wet Medisch-wetenschappelijk Onderzoek met mensen) in particular. This study was approved by the medical ethics committee Stichting het Nederlands Kanker Instituut - Antoni van Leeuwenhoek ziekenhuis Amsterdam, the Netherlands (PTC15.0111/M14CNB).

The study will be performed in 35 participating centres, including 4 university medical centres. The Board of Directors approved initiation of the study in current participating centres that are open for accrual. The BOOG 2013-08 trial is registered at ClinicalTrials.gov (NCT02271828) [30]. All patients will be provided with written information in the form of a Patient Information Folder, including the study aims, randomization procedure, possible AEs, and their rights and responsibilities. Written informed consent must be obtained for all patients included in the study before they are randomized in the study.

\section{Consent for publication}

Not applicable.

\section{Competing interests}

The authors declare that they have no competing interests.

\section{Author details}

'Division of Surgical Oncology, Maastricht University Medical Centre, P.O. Box 58006202 AZ Maastricht, The Netherlands. ${ }^{2}$ GROW - School for Oncology and Developmental Biology, Maastricht University Medical Centre, Maastricht, the Netherlands. ${ }^{3}$ Division of Surgical Oncology, Diakonessenhuis Hospital, Utrecht, the Netherlands. ${ }^{4}$ Division of Surgical Oncology, Netherlands Cancer Institute - Antoni van Leeuwenhoek Hospital, Amsterdam, the Netherlands. ${ }^{5}$ Division of Surgical Oncology, Canisius-Wilhelmina Hospital, Nijmegen, the Netherlands. ${ }^{6}$ Department of Radiation Oncology, Maastricht University Medical Centre (MAASTRO clinic), Maastricht, the Netherlands. 'Division of Medical Oncology, Netherlands Cancer Institute - Antoni van Leeuwenhoek Hospital, Amsterdam, the Netherlands. ${ }^{8}$ Department of Radiology and Nuclear Medicine, Maastricht University Medical Centre, Maastricht, the Netherlands. ${ }^{9}$ Department of Radiation Oncology, Radboud University Medical Centre, Nijmegen, the Netherlands. ${ }^{10}$ Division of Medical Oncology, Maastricht University Medical Centre, Maastricht, the Netherlands.

${ }^{11}$ Department of Pathology, Netherlands Cancer Institute - Antoni van Leeuwenhoek Hospital, Amsterdam, the Netherlands. ${ }^{12}$ Department of Medical and Clinical Psychology, Tilburg University, Tilburg, the Netherlands. ${ }^{13}$ Radiation Oncology, Radiotherapy group, Arnhem, the Netherlands. ${ }^{14}$ Department of Clinical Epidemiology and Medical Technology Assessment, Maastricht University Medical Centre, Maastricht, the Netherlands. ${ }^{15}$ Division of Surgical Oncology, Radboud University Medical Centre, Nijmegen, the Netherlands.
Received: 24 November 2015 Accepted: 22 June 2017

Published online: 01 July 2017

\section{References}

1. Voogd AC, Coebergh JW, Repelaer van Driel OJ, Roumen RM, van Beek MW, Vreugdenhil A, et al. The risk of nodal metastases in breast cancer patients with clinically negative lymph nodes: a population-based analysis. Breast Cancer Res Treat. 2000;62(1):63-9.

2. Krag DN, Anderson SJ, Julian TB, Brown AM, Harlow SP, Ashikaga T, et al. Technical outcomes of sentinel-lymph-node resection and conventional axillary-lymph-node dissection in patients with clinically node-negative breast cancer: results from the NSABP B-32 randomised phase III trial. Lancet Oncol. 2007:8(10):881-8.

3. Peintinger F, Reitsamer R, Stranzl H, Ralph G. Comparison of quality of life and arm complaints after axillary lymph node dissection vs sentinel lymph node biopsy in breast cancer patients. Br J Cancer. 2003;89(4):648-52.

4. Schulze T, Mucke J, Markwardt J, Schlag PM, Bembenek A. Long-term morbidity of patients with early breast cancer after sentinel lymph node biopsy compared to axillary lymph node dissection. J Surg Oncol. 2006; 93(2):109-19.

5. Ohsumi SK S, Takahashi M, Hara F, Takabatake D, Takashima S, Aogi K, et al. Sensory Disturbance of the Ipsilateral Upper Arm after Breast Cancer Surgery with Sentinel Node Biopsy Alone Compared with Axillary Dissection - A Prospective Study. In: 34th Annual San Antonio Breast Cancer Symposium, San Antonio Texas; 2011

6. Lucci A, McCall LM, Beitsch PD, Whitworth PW, Reintgen DS, Blumencranz PW, et al. Surgical complications associated with sentinel lymph node dissection (SLND) plus axillary lymph node dissection compared with SLND alone in the American College of Surgeons Oncology Group Trial Z0011. J Clin Oncol. 2007:25(24):3657-63.

7. Ashikaga T, Krag DN, Land SR, Julian TB, Anderson SJ, Brown AM, et al. Morbidity results from the NSABP B-32 trial comparing sentinel lymph node dissection versus axillary dissection. J Surg Oncol. 2010;102(2):111-8.

8. Fisher B, Anderson S, Bryant J, Margolese RG, Deutsch M, Fisher ER, et al. Twenty-year follow-up of a randomized trial comparing total mastectomy, lumpectomy, and lumpectomy plus irradiation for the treatment of invasive breast cancer. N Engl J Med. 2002;347(16):1233-41.

9. Giuliano AE, Hunt KK, Ballman KV, Beitsch PD, Whitworth PW, Blumencranz PW, et al. Axillary dissection vs no axillary dissection in women with invasive breast cancer and sentinel node metastasis: a randomized clinical trial. JAMA. 2011;305(6):569-75.

10. Galimberti V, Cole BF, Zurrida S, Viale G, Luini A, Veronesi P, et al. Axillary dissection versus no axillary dissection in patients with sentinel-node micrometastases (IBCSG 23-01): a phase 3 randomised controlled trial. Lancet Oncol. 2013;14(4):297-305.

11. Kvistad KA, Rydland J, Smethurst HB, Lundgren S, Fjosne HE, Haraldseth O. Axillary lymph node metastases in breast cancer: preoperative detection with dynamic contrast-enhanced MRI. Eur Radiol. 2000;10(9):1464-71.

12. Nori J, Vanzi E, Bazzocchi M, Bufalini FN, Distante V, Branconi F, et al. Role of axillary ultrasound examination in the selection of breast cancer patients for sentinel node biopsy. Am J Surg. 2007;193(1):16-20.

13. Pamilo M, Soiva M, Lavast EM. Real-time ultrasound, axillary mammography, and clinical examination in the detection of axillary lymph node metastases in breast cancer patients. J Ultrasound Med. 1989;8(3):115-20.

14. Valente SA, Levine GM, Silverstein MJ, Rayhanabad JA, Weng-Grumley JG, Ji $L$, et al. Accuracy of predicting axillary lymph node positivity by physical examination, mammography, ultrasonography, and magnetic resonance imaging. Ann Surg Oncol. 2012;19(6):1825-30.

15. Houssami N, Ciatto S, Turner RM, Cody HS 3rd, Macaskill P. Preoperative ultrasound-guided needle biopsy of axillary nodes in invasive breast cancer: meta-analysis of its accuracy and utility in staging the axilla. Ann Surg. 2011; 254(2):243-51.

16. Neal CH, Daly CP, Nees AV, Helvie MA. Can preoperative axillary US help exclude N2 and N3 metastatic breast cancer? Radiology. 2010;257(2):335-41.

17. Schipper RJ, van Roozendaal LM, de Vries B, Pijnappel RM, Beets-Tan RG, Lobbes MB, et al. Axillary ultrasound for preoperative nodal staging in breast cancer patients: is it of added value? Breast. 2013;22(6):1108-13.

18. van Wely BJ, de Wilt JH, Francissen C, Teerenstra S, Strobbe LJ. Meta-analysis of ultrasound-guided biopsy of suspicious axillary lymph nodes in the selection of patients with extensive axillary tumour burden in breast cancer. Br J Surg. 2015;102(3):159-68 
19. Early Breast Cancer Trialists' Collaborative G, Darby S, McGale P, Correa C Taylor C, Arriagada $R$, et al. Effect of radiotherapy after breast-conserving surgery on 10-year recurrence and 15-year breast cancer death: metaanalysis of individual patient data for 10,801 women in 17 randomised trials. Lancet. 2011;378(9804):1707-16.

20. Rastogi P, Anderson SJ, Bear HD, Geyer CE, Kahlenberg MS, Robidoux A, et al. Preoperative chemotherapy: updates of National Surgical Adjuvant Breast and Bowel Project Protocols B-18 and B-27. J Clin Oncol. 2008;26(5):778-85.

21. Straver ME, Rutgers EJ, Russell NS, Oldenburg HS, Rodenhuis S, Wesseling J, et al. Towards rational axillary treatment in relation to neoadjuvant therapy in breast cancer. Eur J Cancer. 2009;45(13):2284-92.

22. Rouzier R, Extra JM, Klijanienko J, Falcou MC, Asselain B, Vincent-Salomon A, et al. Incidence and prognostic significance of complete axillary downstaging after primary chemotherapy in breast cancer patients with T1 to T3 tumors and cytologically proven axillary metastatic lymph nodes. J Clin Oncol. 2002;20(5):1304-10.

23. Alvarado R, Yi M, Le-Petross H, Gilcrease M, Mittendorf EA, Bedrosian I, et al. The role for sentinel lymph node dissection after neoadjuvant chemotherapy in patients who present with node-positive breast cancer. Ann Surg Oncol. 2012;19(10):3177-84.

24. van Roozendaal LM, Schipper RJ, Van de Vijver KK, Haekens CM, Lobbes MB, Tjan-Heijnen VC, et al. The impact of the pathological lymph node status on adjuvant systemic treatment recommendations in clinically node negative breast cancer patients. Breast Cancer Res Treat. 2014;143(3):469-76.

25. van Wely BJ, Teerenstra S, Schinagl DA, Aufenacker TJ, de Wilt JH, Strobbe $\sqcup$. Systematic review of the effect of external beam radiation therapy to the breast on axillary recurrence after negative sentinel lymph node biopsy. Br J Surg. 2011;98(3):326-33.

26. Reed DR, Lindsley SK, Mann GN, Austin-Seymour M, Korssjoen T, Anderson $\mathrm{BO}$, et al. Axillary lymph node dose with tangential breast irradiation. Int J Radiat Oncol Biol Phys. 2005;61(2):358-64.

27. Chung MA, DiPetrillo T, Hernandez S, Masko G, Wazer D, Cady B. Treatment of the axilla by tangential breast radiotherapy in women with invasive breast cancer. Am J Surg. 2002;184(5):401-2.

28. Rabinovitch R, Ballonoff A, Newman F, Finlayson C. Evaluation of breast sentinel lymph node coverage by standard radiation therapy fields. Int J Radiat Oncol Biol Phys. 2008;70(5):1468-71.

29. van Roozendaal LM, Schipper RJ, Smit LH, Brans BT, Beets-Tan RG, Lobbes $\mathrm{MB}$, et al. Three-dimensional breast radiotherapy and the elective radiation dose at the sentinel lymph node site in breast cancer. Ann Surg Oncol. 2015;22(12):3824-30

30. van Roozendaal LM, de Wilt JH, van Dalen T, van der Hage JA, Strobbe $\sqcup$, Boersma $\sqcup$, et al. The value of completion axillary treatment in sentinel node positive breast cancer patients undergoing a mastectomy: a Dutch randomized controlled multicentre trial (BOOG 2013-07). BMC Cancer. 2015;15:610.

31. Guideline Mammacarcinoma 2012 the Netherlands [http://richtlijnendatabase. $\mathrm{nl} /$ en/richtlijn/breast_cancer/locoregional_treatment.html].

32. Abe H, Schmidt RA, Kulkarni K, Sennett CA, Mueller JS, Newstead GM. Axillary lymph nodes suspicious for breast cancer metastasis: sampling with US-guided 14-gauge core-needle biopsy-clinical experience in 100 patients. Radiology. 2009;250(1):41-9.

33. Koelliker SL, Chung MA, Mainiero MB, Steinhoff MM, Cady B. Axillary lymph nodes: US-guided fine-needle aspiration for initial staging of breast cancercorrelation with primary tumor size. Radiology. 2008;246(1):81-9.

34. Offersen BV, Boersma $\sqcup$, Kirkove C, Hol S, Aznar MC, Biete Sola A, et al. ESTRO consensus guideline on target volume delineation for elective radiation therapy of early stage breast cancer. Radiother Oncol. 2015;114(1):3-10.

35. Aaronson NK, Ahmedzai S, Bergman B, Bullinger M, Cull A, Duez NJ, et al. The European Organization for Research and Treatment of Cancer QLQ-C30: a quality-of-life instrument for use in international clinical trials in oncology. J Natl Cancer Inst. 1993;85(5):365-76.

36. Sprangers MA, Groenvold M, Arraras JI, Franklin J, te Velde A, Muller M, et al. The European Organization for Research and Treatment of Cancer breast cancer-specific quality-of-life questionnaire module: first results from a three-country field study. J Clin Oncol. 1996;14(10):2756-68.

37. Devoogdt N, Van Kampen M, Geraerts I, Coremans T, Christiaens MR. Lymphoedema functioning, disability and health questionnaire (Lymph-ICF): reliability and validity. Phys Ther. 2011;91(6):944-57.
38. van der Bij AK, de Weerd S, Cikot RJ, Steegers EA, Braspenning JC. Validation of the dutch short form of the state scale of the Spielberger State-Trait Anxiety Inventory: considerations for usage in screening outcomes. Community Genet. 2003:6(2):84-7.

39. van der Steeg AF, De Vries J, van der Ent FW, Roukema JA. Personality predicts quality of life six months after the diagnosis and treatment of breast disease. Ann Surg Oncol. 2007;14(2):678-85.

40. Moossdorff M, van Roozendaal LM, Strobbe LJ, Aebi S, Cameron DA, Dixon JM, Giuliano AE, Haffty BG, Hickey BE, Hudis CA et al. Maastricht Delphi consensus on event definitions for classification of recurrence in breast cancer research. J Natl Cancer Inst. 2014;106(12). doi:10.1093/jnci/dju288. Print 2014 Dec.

41. Moossdorff M, van Roozendaal LM, Schipper RJ, Strobbe LJ, Voogd AC, TjanHeijnen VC, et al. Inconsistent selection and definition of local and regional endpoints in breast cancer research. Br J Surg. 2014;101(13):1657-65.

\section{Submit your next manuscript to BioMed Central and we will help you at every step:}

- We accept pre-submission inquiries

- Our selector tool helps you to find the most relevant journal

- We provide round the clock customer support

- Convenient online submission

- Thorough peer review

- Inclusion in PubMed and all major indexing services

- Maximum visibility for your research

Submit your manuscript at www.biomedcentral.com/submit 